\title{
HISTERECTOMÍA MÍNIMAMENTE INVASIVA A TRAVÉS DE LAPAROSCOPIA CON BISTURÍ ULTRASÓNICO
}

\author{
Vicente Solà D. ${ }^{1}$, Jack Pardo S. ${ }^{1}$, Paolo Ricci A. ${ }^{1}$ \\ ${ }^{1}$ Unidad de Ginecología, Departamento de Obstetricia y Ginecología, Clínica Las Condes.
}

\section{RESUMEN}

Objetivo: Revisar la seguridad, eficacia y reproducibilidad de la histerectomía laparoscópica con bisturí ultrasónico. Discutir las ventajas comparativas con las técnicas de electrocirugía. Método: Se realiza un estudio de seguimiento descriptivo de 18 pacientes ingresadas a la Unidad de Ginecología de Clínica Las Condes, con requerimiento de histerectomía. La edad fluctuó entre 38 y 66 años, con una media de 47 años. La paridad media fue de 2 partos. El índice de masa corporal fue de 28 . Se realizaron 5 histerectomías totales laparoscópicas (HTL), 9 supracervicales laparoscópicas (HSL) y 4 laparoscópicas totales concluídas por vía vaginales (HTLCV). En 13 pacientes se asoció otra cirugía. Resultados: El tiempo operatorio para la HSL fue entre 23 y 45 minutos (media $35 \mathrm{~min}$ ), para HTL entre 45 y 90 minutos (65 min), para HTLCV entre 60 y 90 minutos $(75 \mathrm{~min})$. No se registraron complicaciones en el intraoperatorio, postoperatorio inmediato, temprano, ni tardío. La escala visual análoga para dolor fue a las 12 horas de 2 a 5 para HTL, 0 a 2 para HSL, y 2 a 5 para HTLCV; a las 24 horas fue entre 2 y 4 para HTL, entre 0 y 2 para HSL y entre 2 y 5 para HTLCV. Conclusiones: La histerectomía laparoscópica con bisturí ultrasónico es una técnica segura, eficaz y reproducible. La utilización del bisturí ultrasónico en la histerectomía, permite cortar y coagular, con ventajas comparativas sobre la electrocirugía. Permite reducir la posibilidad de daño de tejidos y estructuras vecinas al útero. El bisturí armónico tiene el potencial para convertirse en una de las energías preferidas para la realización de cirugías laparoscópicas.

\section{PALABRAS CLAVES: Bisturí ultrasónico, bisturí armónico, histerectomía laparoscópica, cirugía mínimamente invasiva, laparoscopia}

\section{SUMMARY}

Objective: To evaluate the security, effectiveness and reproducibility of the laparoscopic hysterectomy by ultrasonic scalpel. To discuss the comparatives advantages with the electro surgery technique. Method: A descriptive prospective study about 18 patients underwent laparoscopic hysterectomy with ultrasonic scalpel in Las Condes Clinic. The age fluctuated between 38 and 66 years old (median 47 years old). The median parity was 2 childbirths. The body mass index was 28.5 total laparoscopic hysterectomies (TLH), 9 supracervical laparoscopic hysterectomies (SLH) and 4 total hysterectomies finished by vaginal route (TLHFV) were made. In 13 patients other surgery was associated. Results: The surgical time for SLH was between 23 and 45 minutes (mean 35 minutes), for LTH between 45 and 90 minutes (mean 65 minutes), for TLHFV between 60 and 90 minutes (mean 75 minutes). Complications were not registered during the intraoperative, immediate, early or late postoperative times. The visual analogue scale for pain at 12 hours was 2 to 5 for LTH, 0 to 2 for SLH, and 2 to 5 for TLHFV; at 24 hours was 2 to 4 for HTL, 0 to 2 for SLH and 2 to 5 for TLHFV. Conclusions: The laparoscopic hysterectomy with ultrasonic scalpel is a safe, effective and feasible surgery allows to cut and to coagulate with comparative advantages on electrosurgery. It allows 
reducing the damage of near tissues and organs. The ultrasonic scalpel has the potential to become one of the energies preferred for the laparoscopies surgeries.

\section{KEY WORDS: Ultrasonic scalpel, harmonic scalpel, laparoscopic hysterectomy, minimally invasive surgery, laparoscopy}

\section{INTRODUCCIÓN}

Durante los últimos años la cirugía mínimamente invasiva ha alcanzado un gran desarrollo. Al utilizar una técnica quirúrgica menos invasiva, necesariamente permitirá una recuperación más rápida y con menores posibilidades de complicaciones. La ginecología no ha quedado al margen de este desarrollo. Sin embargo, esto no sería posible sin un desarrollo adecuado de nuevas tecnologías. El desarrollo de la laparoscopia permite realizar histerectomías supracervicales o totales, con mayores ventajas al compararla con la vía abierta abdominal en pacientes seleccionados (1).

Recientemente se ha incorporado a esta técnica, el bisturí ultrasónico (2-4), que presenta ventajas sobre la electrocirugía (5). Al no utilizar calor, permite cortar y coagular por medio de ultrasonido, disminuyendo la posibilidad de dañar estructuras vecinas por disipación de temperatura (6).

Con el objetivo de comprobar la seguridad, eficacia y reproducibilidad de la histerectomía laparoscópica con ultrasonido, se analiza en forma prospectiva nuestra experiencia y se discuten las ventajas comparativas con las técnicas tradicionales.

\section{PACIENTES Y MÉTODOS}

Se realizó un estudio descriptivo prospectivo de 18 pacientes sometidas a histerectomía total o supracervical laparoscópica, entre agosto del 2006 y febrero del 2007, utilizando bisturí ultrasónico Harmonic Ace ${ }^{\circledR}$ (Ethicon Endo-Surgery Inc, Johnson \& Johnson, Cincinnati, Ohio) (Figura 1). Las pacientes correspondían a ingresos de la Unidad de Ginecología de Clínica Las Condes.

En la Tabla I se presentan las características generales de las pacientes estudiadas. La edad fluctuó entre 38 y 66 años, con una media de 47 años. La paridad media fue de 2 partos. El índice de masa corporal fue de 28. La indicación quirúrgica fue por miomas uterinos sintomáticos, adenomiosis y/o prolapso uterino. Se realizaron 5 histerectomías totales laparoscópicas (HTL), 9 supracervicales laparoscópicas (HSL), y 4 laparoscópicas totales concluidas por vía vaginal (HTLCV) 0 tipo 3 según la clasificación de Reich (7) y Mage y cols (8), en la que se realiza la ligadura de las arterias uterinas por laparoscopia y se continúa por vía vaginal. En 13 pacientes se asoció a la histerectomía una reparación de prolapso con malla de anclaje Prolift (9) y/o cirugía de corrección de incontinencia con malla suburetral TVT (10) o TVT-O (11). En todas se usó anestesia general, dosis única de cefazolina 2 gramos endovenosos durante el preoperatorio.

Técnica quirúrgica: Se colocó un movilizador uterino Summy. Insuflación del abdomen con $\mathrm{CO}_{2}$ a través de una aguja de Verres por zona umbilical. Al alcanzar una presión de $15 \mathrm{mmHg}$, se introdujo trocar umbilical 10-12 mm para la cámara. Se realizaron dos punciones suprapúbicas accesorias para inserción de trocares de $5 \mathrm{~mm}$. A través de uno de ellos se introdujo el bisturí ultrasónico, y por el otro una pinza grasper para tracción o una cánula para irrigación-aspiración con suero fisiológico en caso de requerirla.

Técnica quirúrgica específica: Se realizó histerectomía laparoscópica siguiendo tiempos clásicos. En los casos de histerectomía supracervical se cortó con el bisturí ultrasónico a nivel del istmo, en el nacimiento de los ligamentos útero-sacros. El útero se retiró a través de una de las punciones suprapúbicas accesorias, por donde se introdujo un morcelador eléctrico (Kart Storz, Culver City, California) (12). En los casos de histerectomía total, la vagina también se abrió con el bisturí ultrasónico y se retiró el útero por vía vaginal. En la HTLCV se realizó la cirugía por vía laparoscó-



Figura 1. Bisturí ultrasónico Harmonic Ace TM. 
Tabla I

\section{CARACTERÍSTICAS GENERALES DE LAS PACIENTES INGRESADAS AL ESTUDIO}

\author{
Total de pacientes ingresadas \\ Edad \\ Paridad \\ Índice de masa corporal \\ Indicación quirúrgica \\ Histerectomía total laparoscópica \\ Histerectomía supracervical laparoscópica \\ Histerectomía total laparoscópica concluída \\ por vía vaginal \\ Cirugía asociada
}

Pacientes con cirugía asociada

\author{
18 \\ Rango: 38-66 años; Mediana: 47 años \\ Mediana: 2 partos \\ Mediana: 28 \\ Mioma sintomático, adenomiosis, prolapso \\ 5 \\ 9 \\ 4
}

Reparación de prolapso con malla de anclaje Prolift y o corrección de incontinencia con malla suburetral TVT o TVT-O 13 pica hasta la disección de las arterias uterinas, continuando por vía vaginal. Se realizó una colporrafia en sentido longitudinal por vía vaginal con vicryl 2/0 corrido.

Se utilizó analgesia durante el postoperatorio inmediato con ketoprofeno $100 \mathrm{mg}$ endovenoso cada 8 horas, completando tres dosis; luego se indicó ibuprofeno $600 \mathrm{mg}$ cada 12 horas vía oral por 5 a 7 días. La alimentación y deambulación se indicó según cada caso entre las 8 y 12 horas, dependiendo de la cirugía asociada a la histerectomía. El alta hospitalaria se dio entre las 12 y 24 horas dependiendo de la cirugía asociada. La evaluación del dolor postoperatorio se efectuó a través de la aplicación de la escala visual análoga del dolor, a las 12 y 24 horas del postoperatorio.

\section{RESULTADOS}

El tiempo operatorio para la histerectomía supracervical fluctuó entre 23 y 45 minutos, con una media de 35 minutos. Para la histerectomía total el tiempo operatorio fluctuó entre 45 y 90 minutos, con una media de 65 minutos. Para histerectomía total laparoscópica concluída por vía vaginal el tiempo operatorio fluctuó entre 60 y 90 minutos, con una media de 75 minutos. No se registraron complicaciones durante el intraoperatorio, postoperatorio inmediato (primeras 24 horas), temprano (hasta 7 días), ni tardío (después de 7 días). La aplicación de EVA a las 12 horas mostró puntajes entre 2 y 5 para los casos de histerectomía total laparoscópica, entre 0 y 2 para la supracervical laparoscópica, y entre 2 y 5 para la asistida vaginal. A las 24 horas fue entre 2 y 4 para histerectomía total laparoscópica, entre 0 y 2 para la supracervical laparoscópica y entre 2 y 5 para la concluida por vía vaginal.

\section{DISCUSIÓN}

La histerectomía es y seguirá constituyendo una de las principales cirugías en mujeres en edad reproductiva. La laparoscopia constituye una vía que probadamente cumple con el concepto de menor invasión (1). Reich y cols describieron la histerectomía laparoscópica por primera vez en 1989, demostrando las ventajas de la aplicación de esta tecnología (13).

Entre los instrumentos creados durante el último tiempo se encuentra el bisturí armónico. Se trata de un instrumento que genera ultrasonido a partir de energía eléctrica, la que convierte por una cerámica piezoeléctrica, energía mecánica vibratoria. De esta manera es posible producir una vibración en sentido axial, a una frecuencia máxima constante de 55.500 ciclos por segundo (55,5 $\mathrm{KHz}$ ) y a baja temperatura. Con un desplazamiento vertical de 50 a 100 micrones $(14,15)$. La energía eléctrica es convertida en energía mecánica en la pieza de mano, por lo que se asegura que no pasa electricidad a la paciente. Al no alcanzar las altas temperaturas de los instrumentos con energía eléctrica (monopolar o bipolar) y el láser, teóricamente tendremos menos lesiones en los tejidos adyacentes (extensión menor a $1 \mathrm{~mm}$ ) (16) (Figura 2).

Una de las principales ventajas del bisturí armónico, es que permite coagular y cortar tejidos, a través de sus dos efectos ultrasónicos, la "coaptación" y la "cavitación". Además se trata de un instrumento muy versátil, ya que permite cortar, coagular, traccionar y disecar, sin necesidad de cambiar de instrumento.

La coaptación es la transferencia de energía mecánica a los tejidos. Esto provoca una vibración que permite la desnaturalización de proteínas for- 


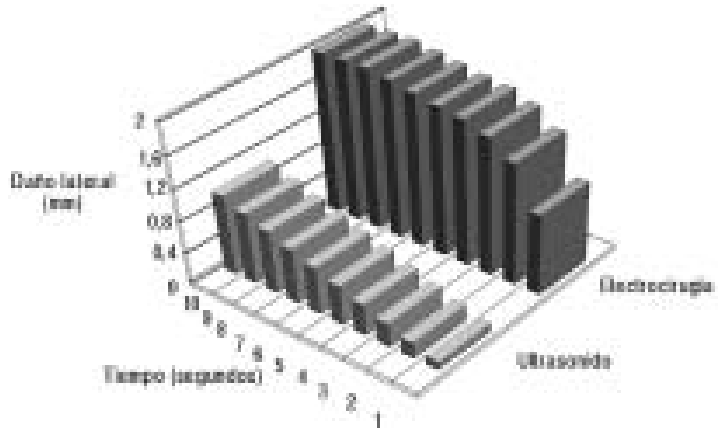

Figura 2. Comparación del daño tisular adyacente al utilizar electro cirugía versus ultrasonido.

mando un coágulo adherente, a través de la ruptura de moléculas de hidrógeno $(17,18)$. En cambio la cavitación es producida por la vaporización de fluidos y ruptura de células. El movimiento de la hoja produce un área de baja presión, causando que los fluidos se vaporicen a baja temperatura. El vapor provoca que las capas de tejido se separen, lo que mejora la visión del campo en la disección, permitiendo al cirujano la localización de los planos. Ambos efectos son rápidos y localizados, evitando la extensión de un posible daño a los tejidos adyacentes.

Entre 50 y 100 grados Celsius, se produce una desorganización proteica por liberación de hidrógeno, formando un coágulo; entre 100 y 150 se produce una vaporización del agua de la célula; entre 150 y 400 grados se produce carbonización (oxidación). El bisturí armónico trabaja entre 50 y 100 grados, lo que constituye la principal diferencia y ventaja comparativa respecto al bisturí eléctrico (monopolar y bipolar) y el láser (ambos producen carbonización/oxidación).

Es muy importante tener en cuenta al momento de utilizar este instrumento, que se requiere un mayor tiempo de aplicación y sin tracción, comparativamente a los instrumentos de energía eléctrica. Este instrumento permite el sellado efectivo de vasos de hasta $5 \mathrm{~mm}$ en un promedio de 4 a 6 segundos. La hoja de bisturí no conduce electricidad y se produce sellado por desnaturalización de proteínas. Actualmente este instrumento se utiliza exitosamente en cirugías como: tiroidectomía, mastectomía, cirugía biliar y hepática, esplenectomía, hemorroidectomía, prostatectomía y cirugía bariática (19-21).

La ventaja de este instrumento es que causa mínimo daño en los tejidos vecinos (22), convirtiéndose en un instrumento de mayor seguridad para el cirujano y la paciente, además de facilitar
Tabla II

VENTAJAS DE LA ENERGÍA ULTRASÓNICA SOBRE LA ELECTROCIRUGÍA

- Mínimo daño térmico de tejidos vecinos

- Instrumento versátil (permite coagular, cortar, traccionar, disecar)

- Control y precisión sobre corte y coagulación

- No carboniza los tejidos

- Baja formación de humo

- No produce dispersión de energía

- No hay paso de electricidad

- No provoca estimulación neuromuscular

la disección cerca de estructuras vitales (10) (Tabla II).

En la casuística presentada hemos podido comprobar las ventajas comentadas al no ver prolongados los tiempos quirúrgicos, no observar complicaciones quirúrgicas y con una excelente recuperación de las pacientes.

\section{CONCLUSIONES}

La histerectomía laparoscópica con bisturí ultrasónico es una técnica segura, eficaz y reproducible. La utilización del bisturí ultrasónico en la histerectomía, permite cortar y coagular, con ventajas comparativas sobre la electrocirugía y el láser. Al utilizar ultrasonido se reduce la posibilidad de daño de tejidos y estructuras vecinas al útero, como vasos sanguíneos, intestino, epiplón y vías urinarias. Al no utilizar energía eléctrica, se evita el paso de corriente a través de la paciente, evitando la lesión de órganos y tejidos fuera de la visión del cirujano. La histerectomía laparoscópica con bisturí ultrasónico requiere de un período breve de entrenamiento y adaptación, para acostumbrarse a los tiempos de aplicación y presión del instrumento durante el corte y coagulación, especialmente de vasos sanguíneos y ligamentos. El bisturí armónico tiene el potencial para convertirse en una de las energías preferidas para la realización de cirugías laparoscópicas.

\section{BIBLIOGRAFÍA}

1. Solà V, Ricci P, Pardo J, Guiloff E. Histerectomía: una mirada desde el suelo pélvico. REV CHIL OBSTET GINECOL 2006;71(5):364-72.

2. Gyr T, Ghezzi F, Arslanagic S, Leidi L, Pastorelli G, Franchi M. Minimal invasive laparoscopic hysterectomy with ultrasonic scalpel. Am J Surg 2001;181(6): 516-9. 
3. Lin J, Zhang X, Xu K. Application of ultrasonic scalpel in gynecologic operative laparoscopy. Chin Med J (Engl) 2001;114(12):1283-5.

4. Kauko M. New techniques using the ultrasonic scalpel in laparoscopic hysterectomy. Curr Opin Obstet Gynecol 1998;10(4):303-5.

5. McCarus SD. Physiologic mechanism of the ultrasonically activated scalpel. J Am Assoc Gynecol Laparosc 1996;3(4):601-8.

6. Kwok A, Nevell D, Ferrier A, Graf N, Lam A, Ford R. Comparison of tissue injury between laparoscopic coagulating shears and electrosurgical scissors in the sheep model. J Am Assoc Gynecol Laparosc 2001;8(3):378-84.

7. Reich $\mathrm{H}$. New techniques in advanced laparoscopic surgery. Baillieres Clin Obstet Gynaecol. 1989;3(3): 655-681

8. Mage G, Wattiez A, Chapron C, Canis M, Pouly JL, Pingeon JM. J Gynecol Obstet Biol Reprod (Paris) 1992;21(4):436-44.

9. Debodinance $\mathrm{P}$, Cosson M, Collinet P, Boukerrou M, Lucot JP, Madi N. Synthetic meshes for transvaginal surgical cure of genital prolapse: evaluation in 2005. J Gynecol Obstet Biol Reprod (Paris) 2006;35(5 Pt 1):429-54.

10. Sola Dalenz V, Pardo Schanz J, Ricci Arriola P, Guiloff Fische E, Chiang Miranda H. TVT associated with other gynecological operations in the same procedure: results and complications. Arch Esp Urol. 2005 Dec;58(10):983-988.

11. Sola Dalenz V, Pardo Schanz J, Ricci Arriola P, Guiloff Fische E, Chiang Miranda H. Minimal invasive surgery in female urinary incontinence: TVT-O. Actas Urol Esp 2006;30(1):61-6.

12. Parekh AR, Moran ME, Newkirk RE, Desai PJ, Calvano CJ. Tissue removal utilizing Steiner Morcellator within a LapSac: effects of a fluid-filled environment. J Endourol. 2000;14(2):185-9.

13. Reich H, DeCaprio J, McGlynn F. Laparoscopic hysterectomy. J Gynecol Surg 1989;5(2):213-6.
14. Amaral JF. Ultrasonic disection. Endosc Surg Allied Technol. 1994;2(3-4):181-185

15. Amaral JF. The experimental development of an ultrasonically activated scalpel for laparoscopic use. Surg Laparosc Endosc 1994;4(2):92-9.

16. McCarus SD. HARMONICTM Ultrasonic Energy in Gynecologic Surgery: Hysterectomy with the HARMONIC ACETM and the McCarus Technique. Hallado en: http://www.obgmanagement.com

17. Grassie K, Makii M. The use of the harmonic scalpel to reduce morbidity during open total abdomina hysterectomies with bilateral salpingo-oophorectomy (TAH/BSO). Curr Surg 2001;58(3):319-22.

18. Stanojevic D, Scepanovic R, Perunovic R, Zivanovic V, Stevanovic P. An ultrasonic scalpel for laparoscopic gynecologic surgery. Srp Arh Celok Lek 1998; 126(5-6):214-6.

19. Takao S, Shinchi H, Maemura K, Aikou T. Ultrasonically activated scalpel is an effective tool for cutting the pancreas in biliary-pancratic surgery: experimental and clinical studies. J Hepatobiliary Pancreat Surg 2000;71(1):58-62.

20. Armstrong DN, Ambroze WL, Schertzer ME, Orangio GR. Harmonic Scalpel vs. electrocautery hemorrhoidectomy: a prospective evaluation. Dis Colon Rectum 2001;44(4):558-64.

21. Targarona EM, Balague C, Marin J, Neto RB, Martinez C, Garriga J, Trias M. Energy sources for laparoscopic colectomy: a prospective randomized comparison of conventional electrosurgery, bipolar computer-controlled electrosurgery and ultrasonic dissection. Operative outcome and costs analysis. Surg Innov 2005;12(4):339-44.

22. Carlander J, Johansson K, Lindstrom S, Velin AK, Jiang $\mathrm{CH}$, Nordborg $\mathrm{C}$. Comparison of experimental nerve injury caused by ultrasonically activated scalpel and electrosurgery. Br J Surg 2005;92(6): 772-7. 